\title{
ON CONVERGENCE OF CLOSED SETS IN A METRIC SPACE AND DISTANCE FUNCTIONS
}

\author{
Gerald BeER
}

\begin{abstract}
Let $\mathrm{CL}(X)$ denote the nonempty closed subsets of a metric space $X$. We answer the following question: in which spaces $X$ is the Kuratowski convergence of a sequence $\left\{C_{n}\right\}$ in $\operatorname{CL}(X)$ to a nonempty closed set $C$ equivalent to the pointwise convergence of the distance functions for the sets in the sequence to the distance function for $C$ ? We also obtain some related results from two general convergence theorems for equicontinuous families of real valued functions regarding the convergence of graphs and epigraphs of functions in the family.
\end{abstract}

Let $\langle X, d\rangle$ be a metric space, and let $\mathrm{CL}(X)$ denote the collection of closed nonempty subsets of $X$. For each $C \in \mathrm{CL}(X)$ the distance function $d(\cdot, C): X \rightarrow[0, \infty)$ for $C$ is defined by the formula $d(x, C)=\inf \{d(x, z): z \in C\}$. In this note we examine the relationship between the convergence of a sequence of such distance functions and the convergence of the sequence of the underlying sets themselves.

DEFINITION. Let $\left\{C_{n}\right\}$ be a sequence of closed sets in a metric space. Then Li $C_{n}$ (respectively Ls $C_{n}$ ) is the set of points $y$ each neighborhood of which meets all but finitely (respectively meets

Received 26 November 1984.

Copyright Clearance Centre, Inc. Serial-fee code: 0004-9727/85 $\$$ A2.00 + 0.00 . 
infinitely) many sets $c_{n}$.

Clearly Li $C_{n} \subset$ Ls $C_{n}$, and both Li $C_{n}$ and Ls $C_{n}$ are closed sets (although possibly. empty). We say $\left\{C_{n}\right\}$ Kuratowski converges [9] to a closed set $C$ if Li $C_{n}=$ Ls $C_{n}=C$; equivalently, Ls $C_{n} \subset C \subset \operatorname{Li} C_{n}$. When $X$ is separable and locally compact, Kuratowski convergence is actually convergence with respect to a certain metrizable topology on $\mathrm{CL}(X) \quad[8]$, but we will proceed more generally here. Suppose $\left\{C, C_{1}, C_{2}, \ldots\right\} \subset \mathrm{CL}(X)$ and $\left\{d\left(\cdot, C_{n}\right)\right\}$ converges pointwise to $d(\cdot, C)$. For each $x$ in $C$ the condition $\lim _{n \rightarrow \infty} d\left(x, C_{n}\right)=0$ implies $\approx \in \operatorname{Li} C_{n}$, so that $C \subset \operatorname{Li} C_{n}$. On the other hand, if $x \in \operatorname{Ls} C_{n}$, there exists a sequence $\left\{x_{k}\right\}$ convergent to $x$ and an increasing sequence of integers $\left\{n_{k}\right\}$ such that, for each $k, x_{k} \in C_{n_{k}}$. We have $\lim _{k \rightarrow \infty} d\left(x, C_{n_{k}}\right)=0$, and it follows from the pointwise convergence of the distance functions that $x \in C$, that is, Ls $C_{n} \subset C$. Thus, pointwise convergence of $\left\{d\left(\cdot, c_{n}\right)\right\}$ to $d(\cdot, C)$ forces Kuratowski convergence of $\left\{C_{n}\right\}$ to $C$. The converse obviously fails.

EXAMPLE 1. Let $X=(0,2)$ as a subspace of the line, and let $c_{n}=(0,1] \cup\{2-(1 / n)\}$. Clearly $\left\{C_{n}\right\}$ Kuratowski converges to $(0,1]$, whereas $\lim _{n \rightarrow \infty} d\left(7 / 4, c_{n}\right)=1 / 4<3 / 4=d(7 / 4,(0,1])$.

What conditions on $X$ ensure the equivalence of the two modes of convergence?

THEOREM 1. Let $\langle X, d\rangle$ be a metric space. The folzowing are equivalent:

(1) whenever $\left\{C_{n}\right\}$ is a sequence in $\mathrm{CL}(X)$ Kuratowski convergent to a closed nonempty set $C$, then $\left\{d\left(\cdot, c_{n}\right)\right\}$ converges pointwise to $d(\cdot, C)$;

(2) for each $p$ in $X$, whenever $\left\{x_{n}\right\}$ is a sequence in $X$ 


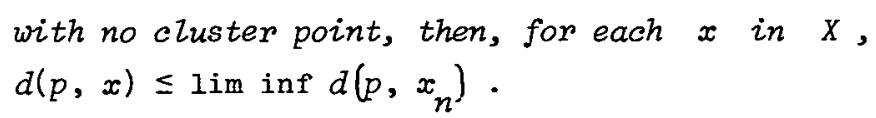

Proof. (1) $\rightarrow(2)$. Suppose (2) fails. Choose points $p$ and $x$ in $X$ and a sequence $\left\{x_{n}\right\}$ in $X$ with no cluster point for which $\lim$ inf $d\left(p, x_{n}\right)<d(p, x)$. By passing to a subsequence we may assume that for some $\varepsilon>0$ and each $n, d\left(p, x_{n}\right)<d(p, x)-\varepsilon$. For each $n$ let $C_{n}=\left\{x, x_{n}\right\}$. Clearly Li $C_{n}=$ Ls $C_{n}=\{x\}$. However, with $C=\{x\}$,

$$
\begin{aligned}
\lim \sup d\left(p, c_{n}\right) & =\lim \sup d\left(p, x_{n}\right) \\
& \leq d(p, x)-\varepsilon \\
& =d(p, c)-\varepsilon .
\end{aligned}
$$

Thus $\left\{d\left(\cdot, c_{n}\right)\right\}$ fails to converge pointwise to $d(\cdot, c)$.

$(2) \rightarrow(1)$. Let $\left\{C_{n}\right\}$ be a sequence in $\operatorname{CL}(X)$ Kuratowski convergent to $C \neq \emptyset$. Fix $p \in X$. Since $C \subset \mathrm{Li} C_{n}$, it is evident that

lim sup $d\left(p, C_{n}\right) \leq d(p, C)$ with no assumptions on $X$. It remains to show $d(p, c) \leq \lim \inf d\left(p, c_{n}\right)$. For each $n$ choose $x_{n} \in C_{n}$ for which $d\left(p, x_{n}\right)<d\left(p, c_{n}\right)+1 / n$. Then there exists a subsequence $\left\{x_{n_{k}}\right\}$ of $\left\{x_{n}\right\}$ for which $\lim _{k \rightarrow \infty} d\left(p, x_{n_{k}}\right\}=\lim$ inf $d\left(p, c_{n}\right)$. If $\left\{x_{n_{k}}\right\}$ has a cluster point $x$, then $x \in$ Ls $C_{n}=C$, and by the continuity of $d$, $d(p, c) \leq d(p, x)=\lim$ inf $d\left(p, c_{n}\right)$. Otherwise, for each $x$ in $X$, $d(p, x) \leq \lim$ inf $d\left(p, x_{n}\right)=\lim$ inf $d\left(p, c_{n}\right)$. In particular, this is true for each $x$ in $c$, whence $d(p, c) \leq \lim$ inf $d\left(p, c_{n}\right)$.

It is easy to check that if $X$ satisfies condition (2) above, then $X$ must be both locally compact and complete. Also, compactness of each closed and bounded subset of $X$ guarantees (2): each sequence $\left\{x_{n}\right\}$ in $X$ without a cluster point eventually lies outside of each ball, whence, for each $p$ in $X$, lim inf $d\left(x_{n}, p\right)=\infty$. The class of spaces for which (2) holds also includes the zero-one metric spaces and others. 
EXAMPLE 2. For each $j \in Z^{+}$let $e_{j}$ be the sequence that takes the value one at $j$ and is zero otherwise. Let $X$ be the metric subspace of $z^{\infty}$ defined by $X=\left\{e_{j}: j \in Z^{+}\right\} \cup\left\{(1 / j) e_{j}: j \in Z^{+}\right\} \cup\{0\}$. For each sequence $\left\{x_{n}\right\}$ in $X$ without a cluster point and for each $p$ in $X$, we have $d\left(p, x_{n}\right)=1$ eventually. Since the diameter of $X$ is one, for each $x$ in $X$ we have $d(p, x) \leq 1=\lim$ inf $d\left(p, x_{n}\right)$. If we replace $\left\{e_{j}: j \in Z^{+}\right\}$in $X$ by $\left\{(j /(j+1)) e_{j}: j \in Z^{+}\right\}$, the space still satisfies condition (2); however, if we replace it by $\left\{((j+1) / j) e_{j}: j \in Z^{+}\right\}$, it no longer does.

Does the pointwise convergence of the distance functions corresponding to a sequence of closed sets bear any relationship to the Kuratowski convergence of the functions, viewed as sets themselves? Most naturally, we can identify a real valued function $f$ on $X$ with its graph in $X \times R$. With this identification, the Kuratowski convergence of a sequence of continuous functions $\left\{f_{n}\right\}$ to a continuous function $f$ can be expressed as follows:

(1) for each $x$ in $X$ there exists $\left\{x_{n}\right\}$ convergent to $x$ for which $f(x)=\lim _{n \rightarrow \infty} f_{n}\left(x_{n}\right)$, and

(2) whenever $\left\{\left(x_{k}, f_{n_{k}}\left(x_{k}\right)\right)\right\}$ converges to $(x, y)$, then $y=f(x)$.

Indeed, condition (1) is equivalent to $f \subset \operatorname{Li} f_{n}$, whereas (2) is equivalent to Ls $f_{n} \subset f$. However, this is not the only identification that can be made, and from the point of view of applications, it is often not the most useful (see, for example, [1] or [11]). As an alternative, we can associate a continuous $f$ with its epigraph, the closed set defined by

$$
\text { epi } f=\{(x, \alpha): \alpha \geq f(x)\} \text {. }
$$

It is an easy exercise to show that Kuratowski convergence of epigraphs is equivalent to the following pair of conditions: 
condition (1) above, and

(2) for each $x$ in $X$, whenever $\left\{x_{n}\right\} \rightarrow x$, then

$$
f(x) \leq \lim \inf f_{n}\left(x_{n}\right)
$$

Of course, continuous functions are not the only ones with closed epigraphs; this larger class of functions is precisely the class of lower semicontinuous functions on $X$ [2]. The study of Kuratowski convergence of epigraphs originated with Wijsman [19], and has its roots in convex analysis. This mode of convergence is now usually called epiconvergence in the literature (see, for example, [10] or [15]).

The distance functions corresponding to a collection of closed sets in a metric space are each Lipschitz with a common Lipschitz constant. Familiar weaker requirements are next described.

DEFINITION. A collection $\Omega$ of real valued functions on a metric space $X$ is called pointwise equicontinuous if for each $p$ in $X$ and $\varepsilon>0$ there exists $\delta>0$, depending on $\varepsilon$ and $p$, such that whenever $d(x, p)<\delta$ then $|f(x)-f(p)|<\varepsilon$ for all $f \in \Omega$. If the same $\delta$ can be chosen for each $p$ in $X$, we call $\Omega$ equicontinuous.

The relationship between pointwise convergence of distance functions and the convergence of distance functions as sets in $X \times R$ falls out of the next theorem.

THEOREM 2. Let $\left\{f_{n}\right\}$ be a pointwise equicontinuous sequence of real valued continuous functions on a metric space $X$, and let $f: X \rightarrow R$ be continuous. The following are equivalent:

(I) whenever $\left\{x_{n}\right\}$ is a sequence in $X$ convergent to $x$, then $\lim _{n \rightarrow \infty} f_{n}\left(x_{n}\right)=f(x)$;

(2) $\left\{f_{n}\right\}$ converges to $f$ uniformly on compact subsets of $X$;

(3) $\left\{f_{n}\right\}$ converges pointwise to $f$;

(4) $\left\{f_{n}\right\}$ Kuratowski converges to $f$;

(5) $\left\{f_{n}\right\}$ epiconverges to $f$. 
Proof. The equivalence of (1) (usually called continuous convergence) and (2) is well known [7], and each condition alone implies pointwise equicontinuity so that this assumption is superfluous here. In view of our local description of (4) and (5), it is evident that (1) implies (3), (4), and (5), again, without an equicontinuity assumption. To see that each of these implies continuous convergence, fix $x$ in $X$ and let $\left\{x_{n}\right\}$ be a sequence convergent to $x$. If any of the last three conditions hold, there exists $\left\{w_{n}\right\}$ convergent to $x$ for which $f(x)=\lim _{n \rightarrow \infty} f_{n}\left(w_{n}\right)$.

Choose $\delta>0$ such that if $d(x, z)<\delta$, then, for each $n$, $\left|f_{n}(x)-f_{n}(z)\right|<\varepsilon / 3$. For all $n$ sufficiently large, we can guarantee that $\left|f_{n}\left(w_{n}\right)-f(x)\right|<\varepsilon / 3, d\left(x_{n}, x\right)<\delta$, and $d\left(w_{n}, x\right)<\delta$, whence $\left|f_{n}\left(x_{n}\right)-f(x)\right|<\varepsilon$.

With no assumptions on the sequence of functions, none of (3), (4), or (5) need imply either of the other two, even if $X$ is compact. It is easy to modify the standard construction [13] of a sequence of nonnegative characteristic functions on $[0,1]$ that converges in measure to the zero function but nowhere converges pointwise to yield a sequence of piecewise linear (continuous) functions with the same property; this sequence will satisfy (5) but neither (3) nor (4). If $f_{n}:[0,1] \rightarrow R$ has as its graph the line segments joining $(0,0)$ to $(1 / 2 n,-1),(1 / 2 n,-1)$ to $(1 / n, 0)$, and $(1 / n, 0)$ to $(1,0)$, then $\left\{f_{n}\right\}$ satisfies (3) but neither (4) nor (5). To see that (4) may hold without either (3) or (5), let $X=\{0\} \cup\left\{1 / k: k \in Z^{+}\right\}$as a subspace of the line, and for each $n \in Z^{+}$define $f_{n}: X \rightarrow R$ to be $-n$ times the characteristic function of $\{0\} \cup\{1 / k: k \geq n\}$. Note that the only point at which $\left\{f_{n}\right\}$ fails to converge is $x=0$. However, one can produce a sequence $\left\{f_{n}\right\}$ on the Cantor set that satisfies (4) but converges nowhere pointwise [5]. One inight guess that if $X$ were connected, then (4) would force (3) and/or (5), but Example 1 of [5] shows this need not occur. What is needed is local connectedness. With this assumption on $X$, (4) not only yields (3) and (5), it also buys (2), and thus (1) [5]. On the other hand, if $X$ is compact and connected, (4) will also buy (1) [4]. Finally, (3) and (5) are equivalent with just a pointwise equi-lower semicontinuity assumption [15]. 
A stronger type of convergence for closed sets is convergence with respect to Hausdorff distance. We pause for a moment to describe this notion. For each point $x$ in $X$ let $B_{\varepsilon}[x]$ denote the closed ball of radius $\varepsilon$ with center $x$, and if $C \in \mathrm{CL}(X)$, denote $\underset{x \in C}{U} B_{\varepsilon}[x]$ by ${ }_{\varepsilon}[C]$. If $K$ is another nonempty closed set, then the Hausdorff distance $H_{d}$ between $C$ and $K$ is given by

$$
H_{d}(C, K)=\inf \left\{\varepsilon: B_{\varepsilon}[C] \supset K \text { and } B_{\varepsilon}[K] \supset C\right\}
$$

The distance $H_{d}$ defines an infinite valued metric on $\mathrm{CL}(X)$. If $d^{\prime}$ is another metric on $X$ which defines the same uniformity (in other words, the identity map from $\langle X, d\rangle$ to $\left\langle X, d^{\prime}\right\rangle$ is bi-uniformly continuous), then $H_{d}$ and $H_{d}$ ' are equivalent on $\mathrm{CL}(X)$. Hausdorff metric convergence always forces Kuratowski convergence in $\mathrm{CL}(X)$; the converse holds precisely in those metric spaces that are compact. For example, if $c_{n}=[0,1] \cup\{n\}$, then $\left\{C_{n}\right\}$ is a Kuratowski convergent sequence in the line which fails to converge in the Hausdorff metric. More facts about Hausdorff distance can be found in [2] and in [6].

Now let $C(X, R)$ denote the continuous real valued functions on $X$. Equip $X \times R$ with the box metric, defined by $\rho\left[\left(x_{1}, \alpha_{1}\right),\left(x_{2}, \alpha_{2}\right)\right]=\max \left\{d\left(x_{1}, x_{2}\right),\left|\alpha_{1}-\alpha_{2}\right|\right\}$. We consider three infinite valued metrics on the function space $C(X, R)$ :

$$
\begin{aligned}
& D_{1}(f, g)=\sup \{|f(x)-g(x)|: x \in X\} ; \\
& D_{2}(f, g)=H_{\rho}(f, g) ; \\
& D_{3}(f, g)=H_{\rho}(\text { epi } f, \text { epi } g) .
\end{aligned}
$$

With no assumptions on $X, D_{2}$ convergence in $C(X, R)$ ensures pointwise convergence, whereas $D_{3}$ convergence does not, even if $X=[0,1]$. Note that metrics equivalent to $D_{2}$ and $D_{3}$ result if we define distance in $x \times R$ to be $\left[d\left(x_{1}, x_{2}\right)^{2}+\left(\alpha_{1}-\alpha_{2}\right)^{2}\right]^{\frac{1}{2}}$, and so on. It is well known [2] that the mapping $C+d(\cdot, C)$ is an isometry from $\left\langle\mathrm{CL}(X), H_{d}\right\rangle$ into 
$\left\langle C(X, R), D_{1}\right\rangle$. Thus Hausdorff metric convergence of a sequence of closed sets in $X$ is equivalent to the uniform convergence of their distance functions.

THEOREM 3. Let $\Omega$ be an equicontinuous family of real functions on a metric space $\langle X, d\rangle$. Then $D_{1}, D_{2}$ and $D_{3}$ are equivalent as infinite valued metrics on $\Omega$.

Proof. We first show that $D_{1}$ is at least as strong as $D_{3}$, in fact, that $D_{3}(f, g) \leq D_{1}(f, g)$. Suppose $D_{1}(f, g)=\lambda$. Let $(x, \alpha)$ be an arbitrary point of epi $f$ and set $\beta=\alpha-f(x)$. Then $(x, g(x)+\beta) \in$ epi $g$, and $\rho[(x, g(x)+\beta),(x, \alpha)]=|g(x)-f(x)| \leq \lambda$. This shows epi $f \subset B_{\lambda}[$ epi $g]$. Similarly, epi $g \subset B_{\lambda}[$ epi $f]$.

We next show that $D_{3}$ is at least as strong as $D_{2}$. Let $\varepsilon$ be positive; we produce $\lambda>0$ such that for all $f, g$ in $\Omega$, $D_{3}(f, g)<\lambda$ implies $D_{2}(f, g) \leq \varepsilon$. Choose $\delta>0$ such that for each $h \in \Omega$ whenever $d(w, z)<\delta$ then $|h(w)-h(z)|<\varepsilon / 3$, and set $\lambda=(1 / 2) \min \{\delta, \varepsilon / 3\}$. Suppose $D_{3}(f, g)<\lambda$ but $D_{2}(f, g)>\varepsilon$. Without loss of generality we can assume there exists $x$ for which $(x, f(x)) \notin B_{\varepsilon}[g]$. Choose $(z, \alpha) \in$ epi $g$ for which $\rho[(z, \alpha),(x, f(x))]<\lambda$. We must have $g(z)<f(x)-\varepsilon$, or else $\rho[(z, g(z)),(x, f(x))] \leq \varepsilon$. Since $\lambda<\delta$, we have $g(x)<(f(x)-\varepsilon)+\varepsilon / 3=f(x)-(2 / 3) \varepsilon$. For the same reason whenever $d(x, w) \leq \lambda$, we have $f(w)>f(x)-\varepsilon / 3$. Hence, if $d(x, w) \leq \lambda$ and $(w, \theta) \in$ epi $f$, we obtain

$$
\begin{aligned}
\rho[(x, g(x)),(w, \theta)] & =\max \{d(x, w), \theta-g(x)\} \\
& \geq \max \{d(x, w), \varepsilon / 3\} \\
& =\varepsilon / 3>\lambda .
\end{aligned}
$$

We have shown $(x, g(x)) \notin B_{\lambda}[$ epi $f]$, in violation of $D_{3}(f, g)<\lambda$.

Finally we show that $D_{2}$ is at least as strong as $D_{1}$. Let $\varepsilon$ be positive, and choose $\delta>0$ such that for each $h$ in $\Omega$, whenever $d(z, w)<\delta$ then $|h(z)-h(w)|<\varepsilon / 2$. Set $\lambda=(1 / 2) \min \{\delta, \varepsilon\}$. Suppose $D_{2}(f, g)<\lambda$ and $x \in X$ is arbitrary. There exists $w \in X$ for which 
both $d(x, w)<\lambda$ and $|f(x)-g(w)|<\lambda$. Since $\lambda<\delta$ we get $|g(x)-g(w)|<\varepsilon / 2$, whence $|f(x)-g(x)|<\lambda+\varepsilon / 2 \leq \varepsilon$. As a result, $D_{1}(f, g) \leq \varepsilon$.

COROLLARY. Let $\langle X, d\rangle$ be a metric space, and let $\left\{C, C_{1}, C_{2}, \ldots\right\} \subset \mathrm{CL}(X)$. Let $\rho$ be a metric on $X \times R$ compatible with the product zoniformity. The following are equivalent:

(1) $\left\{C_{n}\right\}$ converges to $C$ with respect to $H_{d}$;

(2) $\left\{d\left(\cdot, C_{n}\right)\right\}$ converges to $\{d(\cdot, C)\}$ with respect to $H_{\rho}$;

(3) $\left\{\right.$ epi $\left.d\left(\cdot, C_{n}\right)\right\}$ converges to $\{$ epi $d(\cdot, C)\}$ with respect to $H_{p}$.

Proof. The family $\left\{d\left(\cdot, C_{n}\right): n \in Z^{+}\right\} \cup\{d(\cdot, C)\}$ is equicontinuous, and the box metric is compatible with the product uniformity. Any other such metric, by definition, is uniformly equivalent to the box metric, and thus determines the same Hausdorff metric topology on $\mathrm{CL}(X \times R)$.

We note that the proof of Theorem 3 shows that $D_{1}, D_{2}$, and $D_{3}$ are uniformly equivalent metrics on each equicontinuous family $\Omega$. With no assumptions on $\Omega, D_{1}$ is at least as strong as $D_{2}$, and $D_{2}$ is at least as strong as $D_{3}$. If $\Omega$ is merely pointwise equicontinuous, $D_{2}$ may be weaker than $D_{1}$, and $D_{3}$ may be weaker than $D_{2}$.

EXAMPLE 3. Let $X=\{n+(1 / n): n=2,3,4, \ldots\} \cup Z^{+}$, as a subspace of the line. Let $f=x_{z^{+}}$. If, for each $n>1, f_{n}: X \rightarrow R$ is defined by

$$
f_{n}(x)= \begin{cases}1 & \text { if } x=n+1 / n, \\ 0 & \text { if } x=n, \\ f(x) & \text { otherwise, }\end{cases}
$$

then $D_{2}\left(f_{n}, f\right)=1 / n$. However, for each $n, D_{1}\left(f_{n}, f\right)=1$. Instead, if $g$ is the zero function on $X$ and for each $n>1, g_{n}=\chi_{\{n\}}$, then 
$D_{3}\left(g_{n}, g\right)=1 / n$ whereas $D_{2}\left(g_{n}, g\right)=1$. Clearly $\left\{f, f_{2}, f_{3}, \ldots\right\}$ and $\left\{g, g_{2}, g_{3}, \ldots\right\}$ are both pointwise equicontinuous families.

If $X$ is compact, then the proof of $(2) \rightarrow(1)$ in Theorem 3 can be adapted to show that $D_{2}$-convergence in $C(X, R)$ forces $D_{1}$-convergence. Thus, in this case, $D_{1}$ and $D_{2}$ are equivalent (but not uniformly equivalent) finite valued metrics [12]. In fact, if $X$ has finitely many components, the equivalence of $D_{1}$ and $D_{2}$ on $C(X, R)$ characterizes compactness for the domain [4]. A comprehensive study of the approximation of functions with respect to $D_{2}$ has been underway for over twenty years by Sendov, Popov, Veselinov and their associates in Sofia (see, for example, [16] or [17]), whereas this author has produced analogs of some of the fundamental approximation theorems of Stone [18] with respect to $D_{3}$ (see, for example, [3]).

In closing we mention that the convergence of sequences of closed convex sets in a normed linear space and their associated support functionals has been particularly well studied (see [14] and the references therein).

\section{References}

[1] H. Attouch, Variational convergence for functions and operators (Pitman, Marshfield, Massachusetts, 1984).

[2] J.P. Aubin, Applied abstract analysis (John Wiley \& Sons, New York, London, 1977) .

[3] Gerald Beer, "Cone lattices of upper semicontinuous functions", Proc. Amer. Math. Soc. 86 (1982), 81-84.

[4] Gerald Beer, "On uniform convergence of continuous functions and topological convergence of sets", Conad. Math. Bull. 26 (1983), $418-424$.

[5] Gerald Beer, "More on convergence of continuous functions and topological convergence of sets", Canad. Math. Bulz. 28 (1985), 52-59. 
[6] Ch. Castaing and M. Valadier, Convex analysis and measurable multifunctions (Springer-Verlag, Berlin, Heidelberg, New York, 1977).

[1] James Dugundji, Topology (Allyn and Bacon, Boston, 1966).

[8] W. Hildenbrand, Core and equilibria of a large economy (Princeton University Press, Princeton, New Jersey, 1974).

[9] K. Kuratowski, Topology $I$, new edition, revised and augmented (translated by J. Jaworowski. Academic Press, New York and London; Panstwowe Wydawnictwo Naukowe, Warsaw; 1966).

[10] L. McLinden, "Successive approximation and linear stability involving convergent sequences of optimization problems", J. Approx. Theory 35 (1982), 311-354.

[11] L. McLinden and R. Bergstrom, "Preservation of convergence of convex sets and functions in finite dimensions", Trans. Amer. Math. Soc. 268 (1981), 127-142.

[12] S. Naimpally, "Graph topology for function spaces", Trans. Amer. Math. Soc. 123 (1966), 267-272.

[13] R. Royden, Real analysis (Macmillan, New York and London, 1963).

[14] G. Salinetti and R. Wets, "On the convergence of sequences of convex sets in finite dimensions", SIAM Rev. 21 (1979), 18-33.

[15] G. Salinetti, R. Wets and S. Dolecki, "Convergence of functions: equi-semicontinuity", Trons. Amer. Math. Soc. 276 (1983), 409-429.

[16] BI. Sendov and B. Penkov, "Hausdorffsche metrik und approximationen", Numer. Math. 9 (1966), 214-226.

[17] BI. Sendov and V. Popov, "On a generalization of Jackson's theorem for best approximation", J. Approx. Theory 9 (1973), 102-111.

[18] M.H. Stone, "A generalized Weierstrass approximation theorem", Studies in modem analysis, 30-87 (Studies in Mathematics, 1. The Mathematical Association of America, New York, 1962). 
[19] R.A. Wijsman, "Convergence of sequences of convex sets, cones, and functions, II", Trans. Amer. Math. Soc. 123 (1966), 32-45.

Department of Mathematics,

Cal ifornia State University at Los Angeles,

Los Angeles,

Cal ifornia 90032,

USA. 